\title{
Atenção humanizada ao recém-nascido de baixo-peso. Método Canguru e cuidado centrado na família: correspondências e especificidades*
}

I ${ }^{1}$ Marcia de Abreu e Silva Hennig, ${ }^{2}$ Maria Auxiliadora de Souza

Mendes Gomes, ${ }^{3}$ Denise Streit Morsch I

Resumo: Este artigo apresenta a Atenção

Humanizada ao Recém-Nascido de Baixo-Peso -

Método Canguru - como uma diretriz clínica na

assistência neonatal, e busca traçar um paralelo

com outro importante componente do cuidado

infantil descrito na literatura internacional: o

cuidado centrado na família. $\mathrm{O}$ artigo propóe que

a Atenção Humanizada ao Recém-Nascido de

Baixo-Peso - Método Canguru seja vista como

uma boa prática e seja considerada também no

campo das tecnologias em saúde.

> Palavras-chave: Método Canguru; Cuidado

Centrado na Família; recém-nascido de baixo-peso;

diretrizes clínicas; tecnologias em saúde; boas práticas.

\author{
${ }^{1}$ Hospital Central da Polícia \\ Militar (HCPM/PMERJ). \\ Endereço eletrônico: mabreu@ \\ rio.com.br \\ 2 Instituto Fernandes Figueira \\ (IFF). Endereço eletrônico: \\ maria@iff.fiocruz.br \\ ${ }^{3}$ Instituto Fernandes Figueira \\ (IFF). Endereço eletrônico: \\ dmorsch@globo.com
}

Recebido em: 22/10/2009. Aprovado em: 30/04/2010.

* Este artigo é parte da tese de doutorado da primeira autora, pela Pós-Graduação em Saúde da Criança e da Mulher (PGSCM) do Instituto Fernandes Figueira (Fiocruz). Este estudo não recebeu financiamento e não possui nenhum conflito de interesse envolvido. 
Componente integrante da taxa de mortalidade infantil, a mortalidade neonatal reflete a assistência à saúde associada aos fatores biológicos e à assistência pré-natal, ao parto e ao recém-nascido (BN). Assim, assistência materna e neonatal de alto risco inclui investimentos de serviços hospitalares de alta complexidade, que abarquem as necessidades desta área. No Brasil, a implantação de novas tecnologias e práticas, bem como a produção de novos conhecimentos, refletiram significativamente na redução das taxas de mortalidade perinatal, provocando uma ampla mudança na forma de cuidar os recém-nascidos (GOMES, 2004).

Em paralelo aos novos procedimentos clínicos no cuidado ao RN, os estudos voltados para o desenvolvimento cerebral e psíquico desses recém-nascidos vêm apontando para relevância do cuidado em dois âmbitos: ambiental - que comporta as condições de conforto e estética das instalações e equipamentos no local onde a prestação ocorre (AZOULAY; SPRUNG, 2004; GOLDSON, 1999) e relacional - que abarca as relaçóes interpessoais entre os profissionais e o paciente. Ambos estão associados ao cuidado clínico e valorizam a presença da família no cuidado com o recém nascido.

Em estudo anterior (HENNIG et al., 2006), buscamos identificar as rotinas, os conhecimentos e as práticas dos profissionais de maternidades públicas no Município do Rio de Janeiro sobre aspectos selecionados do cuidado neonatal na perspectiva da Atenção Humanizada ao Recém-Nascido de Baixo-pesoMétodo Canguru (AHRNBP-MC) . Neste trabalho, observamos que, apesar do conhecimento teórico sobre a AHRNBP-MC, os profissionais no entanto não o utilizam plenamente em sua prática clínica, sugerindo que a abrangência dessa forma de cuidado neonatal ainda não está totalmente aplicada.

A AHRNBP-MC é uma política pública de saúde cujo conjunto de intervenções, apesar de ter sido criado segundo os critérios de elaboração de diretrizes clínicas, não é visto pelo próprio Ministério da Saúde como tal, apesar da literatura recente sobre o cuidado neonatal evidenciar a importância crescente dos aspectos ligados à humanização e a participação da família (CONNER; NELSON, 1999; MALUSKY, 2005).

A AHRNBP-MC, normatizada em 1999 pelo Ministéio da Saúde (BRASIL, 2001) tem sua inspiração no Método Mãe-Canguru (MMC) criado em 1978, na Colômbia, a partir de uma situação crítica de superlotação de recém-nascidos prematuros e de baixo-peso e de elevadas taxas de mortalidade perinatal por infecções 
cruzadas causadas e pela ausência de recursos tecnológicos. Diferentemente de sua origem na Colômbia, o Método, no Brasil, não teve como objetivo a substituição da estrutura (equipamentos/recursos humanos) como resposta necessária ao cuidado com o recém-nascido de risco e sim, a adoção deste método como promoção de uma mudança institucional na busca de atenção à saúde centrada na humanização da assistência e no princípio de cidadania da família (BRASIL, 2001).

Entendendo a importância e os desafios da efetiva implementação das práticas preconizadas pela AHRNBP-MC, este estudo visa a estabelecer uma correspondência com o preconizado pelo Cuidado Centrado na Família, cujas propostas são consideradas, na literatura internacional, como uma boa prática no cuidado neonatal (HORBAR et al., 2003) propondo que esta política seja reconhecida no cenário das diretrizes clínicas e das tecnologias em saúde,

\section{Medicina baseada em evidências e a melhoria da qualidade da assistência à saúde}

Hoje é internacionalmente aceito que a implementação de diretrizes para a prevenção, diagnóstico, tratamento e reabilitação de doenças definidas a partir da evidência científica disponível acerca da eficácia e efetividade de intervenções produz melhores resultados na população assistida (GRIMSHAW; RUSSELL, 1993; BODENHEIMER et al., 2002).

Em diversos países do mundo, inúmeras organizações têm-se dedicado à sistematização de evidências científicas e desenvolvimento de diretrizes para a assistência à saúde. Dentre estas organizações, algumas agências governamentais merecem destaque pelo volume e independência na produção científica que ocorre de forma direta ou através do financiamento de projetos. Atuando junto a essas agências ou de forma independente e desempenhando um importante papel, incluem-se também sociedades profissionais, instituiçóes acadêmicas e organizações não governamentais (PORTELA, 2003).

Destaque entre as organizações sem fins lucrativos voltadas para a melhoria da saúde das populações, o Institute Of Medicine (IOM, 2001), publicou o relatório Crossing the Quality Chasm: A New Health System for the 21 $1^{\text {st }}$ Century, elaborado pelo Committee on the Quality of Health Care in America, em que apresenta estratégias para melhoria da qualidade do cuidado nos sistemas de saúde, de forma a atender às demandas do século XXI (quadro 1). 
Regras simples para o sistema de saúde do sécullo 21 (IOM, 2001)

\begin{tabular}{|c|c|}
\hline Abordagem Atual & Nova Regra \\
\hline $\begin{array}{l}\text { O cuidado é baseado principalmente em } \\
\text { visitas }\end{array}$ & $\begin{array}{l}\text { O cuidado é baseado num processo de } \\
\text { assistência contínua }\end{array}$ \\
\hline $\begin{array}{l}\text { A autonomia do profissional conduz a } \\
\text { variabilidade do tratamento }\end{array}$ & $\begin{array}{l}\text { O tratamento é individualizado de acordo } \\
\text { com o paciente }\end{array}$ \\
\hline $\begin{array}{l}\text { Cuidado centrado no controle do } \\
\text { profissional }\end{array}$ & O paciente é a fonte do controle \\
\hline A informação é um registro & $\begin{array}{l}\text { O conhecimento é compartilhado e a } \\
\text { informação flui livremente }\end{array}$ \\
\hline $\begin{array}{l}\text { As tomadas de decisão são baseadas em } \\
\text { treinamento e experiência }\end{array}$ & As decisões são baseadas em evidências \\
\hline $\begin{array}{l}\text { Não causar danos é uma responsabilidade } \\
\text { individual }\end{array}$ & $\begin{array}{l}\text { Segurança é um sistema em que todos } \\
\text { participam }\end{array}$ \\
\hline O sigilo é necessário & Transparência é necessária \\
\hline O sistema reage às necessidades & As necessidades são antecipadas \\
\hline Redução de custo é necessária & Desperdício é continuamente reduzido \\
\hline $\begin{array}{l}\text { Preferência é dada ao papel do profissional } \\
\text { sobre o sistema }\end{array}$ & $\begin{array}{l}\text { Cooperação entre os profissionais é uma } \\
\text { prioridade }\end{array}$ \\
\hline
\end{tabular}

Nas diretrizes propostas para a melhoria da qualidade do cuidado (IOM, 2001), é possível observar a mudança no modo de gestão do cuidado em saúde. Se antes as decisões baseavam-se na prática e na experiência do profissional, agora são baseadas em evidências científicas proporcionadas por pesquisas que focalizam a acurácia e precisão de testes diagnósticos, a força de marcadores prognósticos e a eficácia e segurança de protocolos de prevenção, tratamento ou reabilitação.

A medicina baseada em evidências (MBE), entendida como a melhor evidência clínica externa, provinda da pesquisa sistemática, é a principal ferramenta para a implementação das diretrizes clínicas, bem como dos instrumentos de normalização dos processos de trabalho na saúde (MENDES; SHIMAZAKI, 2004). 
As diretrizes clínicas são posicionamentos ou recomendações (statements) desenvolvidos para orientar os profissionais de saúde e pacientes, em circunstâncias clínicas específicas, acerca de cuidados de saúde apropriados (IOM, 1990). As diretrizes constituem-se tanto de indicações/contraindicações, quanto dos benefícios esperados e possibilidade de riscos advindos das tecnologias em saúde (procedimentos, testes diagnósticos, medicamentos, etc) aplicados para grupos de pacientes definidos (PORTELA, 2003).

$\mathrm{Na}$ medida em que as diretrizes se tornam mais sensíveis às reais necessidades dos pacientes, passam a promover participação destes nas decisões que envolvem a escolha das prováveis intervenções determinando, como consequência, sua satisfação com os processos e com os resultados do cuidado recebido. Com isso, as diretrizes auxiliam na identificação de resultados a serem incorporados em pesquisas de satisfação, podendo desempenhar importante papel na detecção de problemas de qualidade na atenção à saúde proposta pelas diferentes instituições. Ou seja, tornando-se potencial de melhora na qualidade da assistência à saúde, as diretrizes clínicas podem contribuir para a determinação de prioridades e padronização de cuidados, além de servirem de indicativos da necessidade de estudos específicos relativos à efetividade de intervençōes (PORTELA, 2003).

O Institute of Medicine (1990, p. 3) definiu qualidade da assistência à saúde como o "grau em que serviços de saúde para individuos e populações melhoram a probabilidade de ocorrência de resultados desejados e consistentes com o conhecimento profissional coerente". Na medida em que as diretrizes se baseiam no conhecimento científico, estimativas dos resultados esperados e julgamento profissional corrente, elas claramente têm um papel na avaliação e garantia de qualidade dos cuidados em saúde.

Por sua vez, os instrumentos de normalização dos processos de trabalho em saúde (MENDES, 2004) constituem recomendações preparadas de forma sistemática e com base em evidências científicas, com o propósito de influenciar decisões a respeito de intervençôes de saúde, ligando medicina baseada em evidência, avaliação tecnológica e garantia de qualidade (EDDY, 1999). Esses instrumentos são divididos, pelos autores, em dois tipos principais: as linhas-guia (guidelines) e os protocolos clínicos.

As linhas-guia, como instrumentos gerenciais, são recomendações que devem gerar uma planilha, cujo principal objetivo é gerir uma atenção apropriada à 
saúde, governando a elaboração da programação dos serviços de saúde em relação a determinada condição ou patologia. Com isso, normaliza todo o processo ao longo de sua história natural, cobre as ações de prevenção primária, secundária, terciária, além de todos os pontos de atenção de um sistema integrado de serviços de saúde (MENDES; SHIMAZAKI, 2004).

Já os protocolos clínicos são recomendações cujo principal objetivo é dirigir uma atenção apropriada, através do detalhamento do diagnóstico e do tratamento, às questôes específicas do processo de saúde-doença.

Depois de elaborados, esses instrumentos de normalização dos processos de trabalho em saúde (linhas-guia e protocolos clínicos) devem ser transformados em produtos educacionais a serem transmitidos aos profissionais e aos usuários do sistema de serviços de saúde. Tanto as diretrizes clínicas quanto os instrumentos de normalização dos processos de trabalho em saúde convergem para o mesmo ponto, isto é, fornecer orientaçooes com base em evidências científicas para a prática clínica, buscando a melhoria da qualidade da assistência.

\section{A AHRNBP-MC como diretriz clínica e instrumento de normalização dos processos de trabalho}

Por ser uma proposta baseada em evidências científicas largamente comprovadas na literatura mundial, a Atenção Humanizada ao Recém-Nascido de Baixo-peso - Método Canguru pode ser considerada tanto uma diretriz clínica quanto um instrumento de normalização de processos de trabalho.

A AHRNBP-MC pode ser considerada uma diretriz clínica na medida em que enfatiza a relevância epidemiológica da condição da gravidez de risco e do recém-nascido também de risco; apresenta potencial de mudança nos resultados de saúde (diminuição das taxas de mortalidade e morbidade neo e perinatal) e requer uso intensivo de tecnologia. Ao mesmo tempo, pode ser considerada também um instrumento de normalização de processos de trabalho, pois indica prioridades na escolha da condição ou patologia de acordo com sua relevância para o sistema de saúde, utilizando os serviços de saúde para a normalização da atenção a condiçôes agudas, em especial, as emergências médicas, opera com a definição de grupo-tarefa multidisciplinar, promove a análise situacional da condiçãoo ou patologia, busca evidências e experiências relevantes, formaliza os instrumentos de normalização, além de publicá-los. Dirigida a uma população 
específica (gestação e neonato de risco), aplicada a partir de um manual elaborado por equipe multidisciplinar de experts e implantada nas unidades após cursos de capacitação de profissionais no método, a AHRNBP-MC contempla essas indicações (BRASIL, 2002). Ao mesmo tempo, contribui para a melhoria da comunicação e dos processos interpessoais entre pacientes e profissionais de saúde - essência da visão humanística de qualidade.

\section{AHRNBP-MC e tecnologias em saúde}

Partindo de um conceito de assistência que não se limita ao conhecimento técnico específico, o processo de transformação e de inovação tecnológica na área da saúde que vem acontecendo nas duas últimas décadas coloca as novas tecnologias médicas à disposição do sistema de saúde, incorporando-as à prática clínica. Como resultado, uma série de modificações importantes - que vão desde mudanças significativas na prática médica cotidiana, nos processos de diagnóstico e terapêutica utilizados nessa prática, até alterações na forma de organização e reorganização dos serviços - vêm acontecendo (VIANNA; CAETANO, 2001).

No âmbito deste processo de transformação e inovação tecnológica na área de saúde, além das várias ferramentas-máquinas, conhecimentos sob a forma de saberes profissionais bem estruturados, como a clínica do médico, da enfermagem, etc., vêm sendo intensamente reconhecidos e mobilizados. Isto permite dizer que há diferentes formas de tecnologia em questão. Essas formas de tecnologia podem ser divididas em: tecnologia dura, que se refere ao instrumental complexo em seu conjunto, englobando todos os equipamentos para tratamentos, exames e a organização de informações; a leve-dura, que se refere aos saberes profissionais como a clínica, a epidemiologia e está inscrita na maneira de organizar sua atuação no processo de trabalho; e uma terceira, chamada de tecnologia leve, que se produz em um processo de relações, isto é, no encontro entre o profissional de saúde e o usuário-paciente (MEHRY, 1999).

Nesse sentido, a AHRNBP-MC pode ser considerada um conjunto de propostas de cuidado que se integra com as diferentes dimensões daquilo que podemos chamar de tecnologia em saúde. Uma destas dimensões é a utilização de procedimentos que implicam o uso de medicamentos, o controle ambiental, a utilização de equipamentos para esse cuidado. Integra também outra dimensão, a da chamada tecnologia leve, isto é, os aspectos relacionais envolvidos no cuidado. 
A tecnologia leve, produzida através de um processo de relações onde um sujeito atua sobre o outro, possibilita que sejam criados momentos de falas, escutas e interpretações, que podem proporcionar momentos de confiabilidade e esperança, gerando relações de vínculo e aceitação (MEHRY, 1999).

Esta tecnologia permeia toda a AHRNBP-MC, uma vez

(...) que requer dos profissionais conhecimentos acerca das particularidades físicas e biológicas e das necessidades especiais de cuidados técnicos e psicológicos da gestante, da mãe, do recém-nascido de baixo-peso e de toda a família. Abrange também a equipe de profissionais responsável por esse atendimento, buscando motivá-la para mudanças importantes em suas ações como cuidadores. (BRASIL, 2002).

\section{AHRNBP-MC e cuidado centrado na família: correspondências e especificidades}

A sobrevida dos recém-nascidos de risco e os aspectos éticos que envolvem o seu cuidado são um desafio que deve ser objeto de reflexão sistemática e abrangente exigindo, portanto, a priorização e urgência na adoção de estratégias que possam garantir o acesso a práticas assistenciais adequadas e baseadas nas melhores evidências possíveis.

Considerando um cuidado que reconhece a importância dos pais na vida dos recém-nascidos e enfatiza o respeito, apoio e parceria entre a família e os profissionais de saúde, a forma de cuidado proposta pela AHRNBP-MC encontra um correspondente, na literatura internacional, denominado Cuidado Centrado na Família (Family-Centered Care - FCC), reconhecido como um fundamental componente do cuidado pediátrico, incluindo o neonatal, que enfatiza a importância de reconhecer e aceitar o apoio familiar no processo saúde-doença (AZOULAY; SPRUNG, 2004; MALUSKY, 2005; HORBAR et al, 2003; HARRISON, 1993).

O Cuidado Centrado na Família (CCF) surgiu nos Estados Unidos na década de 60, sendo inicialmente dirigido às crianças com necessidades especiais e suas famílias e, posteriormente, solicitado por pais e profissionais de saúde envolvidos na atenção aos recém-nascidos gravemente enfermos, nas unidades de terapia intensiva neonatais (HARRISON, 1993; CHIOU; WANG, 2004). Os benefícios advindos do CCF, tais como a redução do estresse, têm sido pesquisados em várias das UTIN participantes da rede multicêntrica de pesquisas em neonatologia, ${ }^{1}$ Vermont Oxford Network (VON) e, a partir de seus resultados, este modelo passou a ser reconhecido como uma Boa Prática (HORBAR, 1999; HORBAR et al., 2003, 2007). 
As Boas Práticas são protocolos que têm como objetivo unificar critérios sobre diferentes temas relativos a medicamentos e definem uma série de pautas através das quais os estudos clínicos podem ser disseminados, implementados, finalizados, auditados, analisados e informados de forma a assegurar sua confiabilidade (OPAS, 2006). No campo da neonatologia, as Boas Práticas, referendadas na Medicina Baseada em Evidências (MBE), abarcam estudos variados em relação às práticas utilizadas em unidades de terapia intensiva neonatais (UTIN) - desde as práticas clínicas (uso do surfactante, controle da infecção hospitalar, prevenção de retinopatia da prematuridade, prevenção de hemorragia craniana, etc.), até aquelas que incorporam a qualidade relacional entre a equipe de saúde e familiares (construção da equipe multidisciplinar colaborativa, cuidado centrado na família).

A fim de tornar possível uma parceria colaborativa entre pais e equipe, o CCF tornou necessária a identificação das dificuldades mais significativas inerentes ao envolvimento dos pais no cuidado de seus bebês. Desta forma, foram desenvolvidos os princípios do CCF na atenção neonatal identificados como sendo os mais importantes para os pais e equipe de saúde: segurança, gentileza, comunicação, informação consistente, educação, meio-ambiente, manejo da dor, participação, proximidade, apoio (CONNER; NELSON, 1999).

A experiência brasileira, apresenta correspondências aos princípios do CCF no âmbito do conceito de práticas potencialmente melhores no cuidado neonatal (Potentially Better Practices - PBP), determinado através dos protocolos de rotinas clínicas fortemente disseminadas pela rede multicêntrica de revisão das práticas assistenciais neonatais (HORBAR, 1999; STEWARD, 2001).

Encontramos na AHRNBP-MC correspondências com o CCF no âmbito da participação ativa da família nos cuidados com o paciente, nos itens comunicação e informação, alívio da dor/cuidados individualizados, participação dos pais nos cuidados, ambiente e seguimento pós-alta. E no que se refere à comunicação e informação, as duas propostas trabalham com essa perspectiva desde a detecção da gestação de risco.

As diretrizes do CCF e da AHRNBP-MC se iniciam a partir da identificação das gestantes com maior risco de recém-nascidos de baixo-peso para que sejam oferecidas informações sobre cuidados médicos específicos e humanizados. No CCF, encontramos uma especificidade relacionada à oportunidade dada aos pais de expressar sua preferência pelo tratamento caso seu bebê seja prematuro extremo ou gravemente doente. 
O CCF é baseado numa comunicação livre, ética e honesta entre pais e profissionais. Da mesma forma, a AHRNBP-MC estimula o livre e precoce acesso dos pais a UTIN, buscando uma boa interação entre família e equipe.

Ainda nesse campo, para o CCF os pais devem ter a sua disposição as mesmas informações médicas, informações sobre as incertezas relativas ao tratamento, acesso ao prontuário e participação nos "rounds". A linguagem deve ser acessível para que sejam capazes de fazer escolhas no tratamento. Esses princípios devem ser entendidos no contexto social e cultural do país de origem composto por vários fatores, dentre os quais podemos destacar os marcos legais e a perspectiva dos direitos individuais.

Considerando as diferenças sociais e culturais, encontramos na AHRNBPMC um princípio correspondente: ênfase na importância das orientações sobre as condições de saúde da criança para a mãe e a família. A norma brasileira também destaca a importância da equipe de saúde em se preocupar com o grau de compreensão que a família tem sobre as informações recebidas.

É importante enfatizar que os aspectos comunicação e informação são evidentemente valorizados na AHRNBP-MC. Encontramos no Manual um tópico exclusivo sobre a comunicação entre a família e a equipe de saúde, que visa a despertar entre os profissionais a preocupação quanto à necessidade de uma boa interação com a família, através da comunicação. Neste segmento, o Manual destaca as dificuldades que podem advir de explicações muito técnicas e mesmo da ausência de informações, valorando a escuta dos pais pela equipe, para que seja possível informar a partir das necessidades apontadas por cada família (BRASIL, 2002, p. 48).

O controle da dor é enfatizado nas duas propostas. Ambas apontam a importância de pais e profissionais reconhecerem e aliviarem a dor dos bebês na UTIN. É consenso na comunidade médica que o recém-nascido é capaz de reconhecer a dor e a responder a estímulos nociceptivos. Esta capacidade, associada a sua reduzida competência para inibir a dor, pode refletir-se negativamente no desenvolvimento do sistema nervoso central, através de alterações orgânicas, fisiológicas e comportamentais. É vasta a literatura sobre a importância e os efeitos da dor no recém-nascido (CHERMONT et al., 2003; BHUTTA; ANAND, 2002; AAP, 2000). Encontramos no Manual uma sessão que aborda de forma abrangente os aspectos relativos aos cuidados no manejo da dor/desconforto do RN (BRASIL, 2002, sessão 14). 
O reconhecimento do impacto da ambiência da terapia intensiva neonatal no desenvolvimento dos bebês tem gerado importante discussão entre especialistas da área (KLAUS; KENNELL, 1995; HOATH, 2001; BHUTTA; ANAND, 2002; ALS et al., 2004). A definição de diretrizes sobre aspectos estruturais e funcionais da UTIN visando a minimizar o impacto negativo de fatores como excesso de estímulos sonoros, de luminosidade, variações térmicas e fatores ligados ao manuseio e posicionamento do bebê, é encontrada de forma significativa nas duas propostas. Nos documentos do CCF, encontramos referência explícita de que pais e profissionais devem trabalhar juntos para garantir um ambiente apropriado para os bebês na UTIN. A AHRNBP-MC, além de valorizar esse item, apontando mudanças ambientais e comportamentais necessárias para maior humanização do atendimento do recém-nascido, aponta essas mesmas necessidades para o acolhimento a família e aos profissionais.

O cuidado com os recém-nascidos não se limita ao período da internação. Em ambas as propostas, são colocadas a importância da continuidade da assistência através do acompanhamento ambulatorial, individualizado e especializado. O risco de alteraçóes no desenvolvimento é largamente descrito na literatura e a deteç̧ão precoce destes fatores confirmam a importância de programas interdisciplinares preventivos de follow up, especialmente no primeiro ano de vida, com o intuito de promover o desenvolvimento da criança e minimizar as possíveis sequelas de saúde do período neonatal (BRASIL, 2001; CHARPAK, 2001; NOBRE et al., 2004; OLIVEIRA, 2002; HOLANDA; SILVA, 2004).

Presentes apenas no CCF, os itens participação nas decisões clínicas e avaliação compartilhada das escolhas terapêuicas nos levaram a pensar se isto se deve às já citadas diferenças culturais entre as duas metodologias.

Existentes apenas na norma da AHRNBP-MC, mas citados em vários estudos desenvolvidos pela VON (SAUNDERS, 2003; MOORE et al., 2003; HORBAR, 2006), o treinamento dos profissionais, o acolhimento à família e o contato pele a pele apontam uma estatégia que apresenta especificidades na qualificação do cuidado pautada na atitude dos profissionais de saúde diante do bebê e de sua família a partir de um conceito de assistência que não se limita ao conhecimento técnico específico, mas que o amplia ao habilitar os profissionais na humanização dos cuidados. e ao garantir a presença materna em tempo integral durante a internação do bebê.

Esta análise está sintetizada no quadro 2 . 


\section{Conclusão}

Hoje há consenso na utilização da AHRNBP-MC e, cada vez mais, um número maior de unidades tem essa referência de boa prática, como uma proposta já implantada. No caso específico do município do Rio de Janeiro, ela é considerada uma diretriz clínica que a própria Secretaria Municipal de Saúde (SMS-RJ) adota para as suas diferentes maternidades (HENNIG et al., 2006).

Desta forma, a AHRNBP-MC, como uma política que implica uma estreita relação entre mãe/pai/bebê e entre estes e a equipe de saúde, traz implícita a incorporação das tecnologias leves em seu cotidiano, representando um desafio importante para o sistema de saúde em geral e para os serviços de neonatologia em particular. Porém, para que o desenvolvimento desta atenção ocorra, são necessárias condiçóes que priorizem o cuidado com a família, a fim de que sejam alcançados resultados satisfatórios e/ou efetividade nas ações propostas pela AHRNBP-MC.

As reflexões sobre a inserção da AHRNBP-MC no cenário das diretrizes clínicas e no campo das tecnologias em saúde, bem como as correspondências encontradas com a literatura sobre o Cuidado Centrado na Família podem ser úteis nas estratégias de disseminação e efetiva incorporação dessas proposições nas práticas neonatais.

\section{Quadro 2: Correspondência entre a AHRNBP-MC** (MS, 2002) e o Cuidado Centrado na Família* (HARRISON, 1993)}

\begin{tabular}{|c|c|c|}
\hline & $\mathrm{CCF}^{*}$ & AHRNBP-MC** \\
\hline 1. Comunicação & $\begin{array}{l}\text { O CCF deverá baseado numa } \\
\text { comunicação livre, ética e } \\
\text { honesta entre pais e profissionais. }\end{array}$ & $\begin{array}{l}\text { Estimular o livre e precoce } \\
\text { acesso dos pais a UTIN, } \\
\text { buscando uma boa interação } \\
\text { entre família e equipe. }\end{array}$ \\
\hline 2. Informação & $\begin{array}{l}\text { Os pais devem ter a sua disposição } \\
\text { as mesmas informaçôes médicas, } \\
\text { informaçōes sobre as incertezas } \\
\text { relativas ao tratamento, acesso } \\
\text { ao prontuário e participação nos } \\
\text { "rounds". A linguagem deve ser } \\
\text { acessível para que sejam capazes } \\
\text { de fazer escolhas no tratamento. }\end{array}$ & $\begin{array}{l}\text { Mãe e a família deverão ser } \\
\text { orientadas sobre as condiçōes de } \\
\text { saúde da criança. } \\
\text { A equipe de saúde deve se } \\
\text { preocupar com o grau de } \\
\text { compreensão que a família tem } \\
\text { sobre as informaçōes recebidas. }\end{array}$ \\
\hline
\end{tabular}




\begin{tabular}{|c|c|c|}
\hline $\begin{array}{l}\text { 3. Participação } \\
\text { nas } \\
\text { decisões } \\
\text { clínicas }\end{array}$ & $\begin{array}{l}\text { Nas decisōes clínicas que } \\
\text { envolvem alto risco de mortalidade } \\
\text { e morbidade, grande sofrimento e/ } \\
\text { ou controvérsia no tratamento, os } \\
\text { pais devem ser informados sobre } \\
\text { os benefícios e malefícios relativos } \\
\text { ao tratamento proposto e devem } \\
\text { decidir se concordam ou não com } \\
\text { a terapia proposta. }\end{array}$ & \\
\hline $\begin{array}{l}\text { 4. Gravidez de } \\
\text { risco }\end{array}$ & $\begin{array}{l}\text { Devem ser oferecidas aos } \\
\text { pais informaçôes sobre as } \\
\text { consequências de uma gravidez } \\
\text { de risco. Os pais devem ter a } \\
\text { oportunidade de expressar sua } \\
\text { preferência pelo tratamento caso } \\
\text { seu bebê seja prematuro extremo } \\
\text { ou gravemente doente. }\end{array}$ & $\begin{array}{l}\text { Identificação das gestantes com } \\
\text { maior risco de recém-nascidos } \\
\text { de baixo-peso para que sejam } \\
\text { oferecidas informaçōes sobre } \\
\text { cuidados médicos específicos e } \\
\text { humanizados. }\end{array}$ \\
\hline $\begin{array}{l}\text { 5. Alívio da } \\
\text { dor/cuidados } \\
\text { individualizados }\end{array}$ & $\begin{array}{l}\text { Pais e profissionais devem } \\
\text { reconhecer e aliviar a dor dos } \\
\text { bebês na UTIN. }\end{array}$ & $\begin{array}{l}\text { Profissionais devem propiciar } \\
\text { aos pais conhecimentos sobre os } \\
\text { sistemas de regulação do bebê e } \\
\text { alívio da dor. }\end{array}$ \\
\hline 6. Ambiente & $\begin{array}{l}\text { Pais e profissionais devem } \\
\text { trabalhar juntos para garantir } \\
\text { um ambiente apropriado para os } \\
\text { bebês na UTIN. }\end{array}$ & $\begin{array}{l}\text { Estabelecer uma contínua } \\
\text { adequação tanto da abordagem } \\
\text { técnica quanto das posturas } \\
\text { que impliquem mudanças } \\
\text { ambientais e comportamentais } \\
\text { com vistas à maior } \\
\text { humanização do atendimento. }\end{array}$ \\
\hline $\begin{array}{l}\text { 7. Participação } \\
\text { dos } \\
\text { pais no cuidado }\end{array}$ & $\begin{array}{l}\text { Pais e profissionais trabalham } \\
\text { juntos para assegurar a eficiência } \\
\text { do cuidado neonatal. } \\
\text { Pais e profissionais devem } \\
\text { desenvolver condutas de promoção } \\
\text { de cuidados de maternagem } \\
\text { e encorajar ao máximo o } \\
\text { envolvimento das famílias com } \\
\text { seus bebês hospitalizados. }\end{array}$ & $\begin{array}{l}\text { Valorização da família na } \\
\text { recuperação do bebê. } \\
\text { Estimular que os pais } \\
\text { participem dos cuidados } \\
\text { dispensados ao bebê. }\end{array}$ \\
\hline
\end{tabular}




\begin{tabular}{|c|c|c|}
\hline $\begin{array}{l}\text { 9. Seguimento } \\
\text { pós-alta }\end{array}$ & $\begin{array}{l}\text { Pais e profissionais devem } \\
\text { promover o acompanhamento, a } \\
\text { longo prazo, dos bebês de risco. }\end{array}$ & $\begin{array}{l}\text { Melhorar o prognóstico do } \\
\text { recém-nascido de baixo-peso, } \\
\text { acompanhando a evolução } \\
\text { de seu desenvolvimento } \\
\text { físico e psíquico e } \\
\text { identificar a necessidade de } \\
\text { acompanhamento especializado. }\end{array}$ \\
\hline $\begin{array}{l}\text { 10. Avaliação } \\
\text { compartilhada } \\
\text { das escolhas } \\
\text { terapêuticas }\end{array}$ & $\begin{array}{l}\text { Pais e profissionais devem ter } \\
\text { clareza sobre os danos advindos } \\
\text { tanto da falta quanto do excesso } \\
\text { de procedimentos realizados no } \\
\text { bebê que está em situação crítica, } \\
\text { assim como devem promover } \\
\text { a consciência das necessidades } \\
\text { dos bebês que sobrevivem com } \\
\text { deficiências e assegurar adequado } \\
\text { suporte para eles e suas famílias. }\end{array}$ & \\
\hline $\begin{array}{l}\text { 11. Treinamento } \\
\text { dos profissionais }\end{array}$ & & $\begin{array}{l}\text { Habilitar os profissionais na } \\
\text { humanização dos cuidados } \\
\text { hospitalares e ambientais } \\
\text { considerando a aproximação o } \\
\text { mais precoce possível, entre a } \\
\text { mãe e o bebê, para fortalecer o } \\
\text { vínculo afetivo. }\end{array}$ \\
\hline $\begin{array}{l}\text { 12. Acolhimento } \\
\text { à família }\end{array}$ & & $\begin{array}{l}\text { Incorporar o acolhimento } \\
\text { à família do bebê como } \\
\text { tecnologia necessária para a } \\
\text { assistência ao recém-nascido } \\
\text { internado na UTIN. }\end{array}$ \\
\hline $\begin{array}{l}\text { 13. Contato } \\
\text { pele a pele }\end{array}$ & & $\begin{array}{l}\text { Promover o mais precocemente } \\
\text { possível, o contato pele a pele } \\
\text { entre mãe e bebê, realizado de } \\
\text { maneira orientada, por livre } \\
\text { escolha, de forma crescente e } \\
\text { segura e acompanhado de suporte } \\
\text { assistencial pela equipe de saúde } \\
\text { adequadamente treinada. }\end{array}$ \\
\hline $\begin{array}{l}\text { 14. Garantia da } \\
\text { presença } \\
\text { materna }\end{array}$ & & $\begin{array}{l}\text { Permanência assegurada à mãe, } \\
\text { com suporte assistencial, durante } \\
\text { o período da internação do bebê. }\end{array}$ \\
\hline
\end{tabular}




\section{Referências}

AAP. Committee on Fetus and Newborn; Committee on Drugs; Section on Anestesiology; Section on Surgery; Canadian Paediatric Society. Fetus and Newborn Committee. Prevention and management of pain and stress in neonate. Pediatrics, v. 105, p. 454-461, 2000.

ALS, H. et al. 2004. Early experience alters brain function and strutcture. Pediatrics, v. 113, n. 4, p. 846-857.

AZOULAY, E.; SPRUNG, C.L. Family-physician interactions in the intensive care unit. Crit. Care Med. Nov, v. 32, n. 11, p. 2323-8, 2004.

BODENHEIMER, T.; WAGNER, E.H.; GRUMBACH, K. Improving primary care for patients with chronic illness. JAMA, v. 288, p. 1775-9, 2002.

BRASIL. Ministério da Saúde. Normatização do método Mãe-Canguru. Brasília: MS, 2001. BRASIL. Ministério da Saúde. Atenção Humanizada ao Recém-Nascido de Baixo-peso-Método Mãe-Canguru. Manual Técnico. Brasília: MS, 2002.

BHUTTA, A.T.; ANAND, K.J.S. Vulnerability of the development brain Neuronal mechanisms. Clinics in Perinatology, v. 29, p. 357-372, 2002.

CHARPAK, N. Kangaroo mother care in developing countries. $5^{\text {th }}$ World Congress of Perinatal Medicine, p. 1023-1027, 2001.

CHERMONT, A.G. et al. O que os pediatras conhecem sobre avaliação e tratamento da dor no recém-nascido? Jornal de Pediatria, v. 79, n. 3, p. 265-272, 2003.

CHIOU, C.J.; WANG, H.H. Reflections on the essence of FCC (article in Chinese). Hu Li Za Zi, v. 51, n. 3, p. 53-8, Jun 2004.

CONNER, J.M.; NELSON, E.C. Neonatal Intensive Care: satisfaction measured from a parent's perspective. Pediatrics, v. 103, n. 1, p. 336-349, Jan 1999.

EDDY, D. Practice Policies, what are they? JAMA, n. 263, p. 877-880, 1999.

GOLDSON, E. Nurturing The Premature Infant. Developmental Interventions in the Neonatal Intensive Care Unit. New York: Oxford University Press, 1999.

GOMES, M.A.S.M. O recém-nascido de alto risco: teoria e prática do cuidar. Organização da Assistência Perinatal no Brasil. In: MOREIRA, M.E.L.; LOPES, J.M.A.; CARVALHO, M. (Org). Coleção Criança, Mulher e Saúde. Rio de Janeiro: Fiocruz, 2004.

GRIMSHAW, J.M.; RUSSELL, I.T. Effect of clinical guidelines on medical practice: a systematic review of rigorous evaluation. Lancet, n. 342, p. 1317-1322, 1993.

HARRISON, H. The Principles for Family-Centered Neonatal Care. Pediatrics, v. 92, n. 5, p. 643-650, Nov 1993.

HENNIG, M.A.S.; GOMES, M.A.S.M.; GIANINI, N.O. Conhecimentos e Práticas dos Profissionais de Saúde sobre a "Atenção Humanizada ao Recém-Nascido de Baixo- 
Peso - Método Canguru". Revista Brasileira de Saúde Materno Infantil. Recife, v. 6, n. 4, p. 427-435, 2006.

HOATH, S.B. The skin as a neurodevelopmental interface. NeoReviews, v. 2, n. 12, p. $292-$ 301, 2001.

HOLANDA, A.C.O.S.; SILVA, M.G.C. Caracterização dos recém-nascidos de muito baixo-peso egressos de uma UTI neonatal. Revista Paulista de Pediatria, v. 22, n. 37, 2004.

HORBAR, J.D. The Vermont Oxford Network: Evidence-Based Quality Improvement for Neonatology. Pediatrics, v. 103, n. 1, p. 350-360, Jane 1999.

HORBAR, J.D.; PLSEK, P.E.; LEAHY, K. NIC/Q2000: Establishing Habits for Improvement in NICU. Pediatrics, v. 111, n. 4, p. 397-410, Jan. 2003.

HORBAR, J.D.; PLSEK, P.E.; LEAHY, K. Evidence-Based Quality Improvement in Neonatal and Perinatal Medicine: The Neonatal Intensive Care Quality Improvement Collaborative Experience. Pediatrics, v. 118, p. S57-S64, 2006.

INSTITUTE OF MEDICINE (US). Committee to Advise the Public Health Service on Clinical Practice Guidelines. Clinical Practices Guidelines: Directions for a New Program. Washington, DC: National Academy Press, 1990.

INSTITUTE OF MEDICINE (US). Committee on the Quality of Health Care in America. Crossing the Quality Chasm: A New Health System for the $21^{\text {st }}$ Century, 2001. Disponível em: <http://www.nap.edu/catalog/10027.html>. Acesso em: 20 fev. 2008.

KLAUS, M.H.; KENNELL, J.H. Assistência aos pais. In: . Alto risco em neonatologia. 4a. Edição. Rio de Janeiro: Guanabara Koogan, 1995. p. 139-155.

LATOUR, J.M.; HAZELZET, J.A.; VAN DER HEIJDEN, A.J. Parent satisfaction in pediatric intensive care: a critical appraisal of the literature. Pediatr Crit Care Med., v. 6, n. 5, p. 578-84, Sept 2005.

MALUSKY, S.K. A concept analysis of family-centered care in the NICU. Neonatal Netw, v. 24, n. 6, p. 25-32, Nov/Dec 2005.

MEHRY, E.E. O ato de governar as tensões constitutivas do agir em saúde como desafio permanente de algumas estratégias gerenciais. Ciência e Saúde Coletiva, v. 4, n. 2, p. 305-314, 1999. MENDES, E.V. Os Instrumentos de normalização dos Processos de Trabalho em Saúde. In: - A Gestão da Clínica nos Sistemas de Serviços de Saúde: Minas Gerais. Secretaria de Estado de Saúde de Minas Gerais; 2004, p. 20.

MENDES, E.V.; SHIMAZAKI, M.E. A Gestão da Clinica nos Sistemas de Serviços de Saúde. Belo Horizonte: Secretaria de Estado de Saúde de Minas Gerais, 2004.

MOORE, K.A.C. et al. Implementing potentially better practices for improving familycentered care in neonatal intensive care units: successes and chalenges. Pediatrics, v. III, n. 4, p. e450-e460, 2003. 
NOBRE, F.D.A. et al. Risco neonatal e desenvolvimento infantil avaliado através da "escala de desenvolvimento do comportamento da criança: $1^{\circ}$ ano de vida (EDCC). Revista Paulista de Pediatria, v. 22, p. 35, 2004.

OLIVEIRA, M.C.B. Avaliação do crescimento de recém-nascidos de risco egressos de UTIs neonatais: um estudo prospectivo e multicêntrico. Dissertação (Mestrado) - Instituto Fernandes Figueira, Fiocruz, Rio de Janeiro, 2002.

OPAS. Buenas Práticas Clínicas: Documento de Las Américas. Washington, D.C.: OPS, 2006. PORTELA, M.C. Diretrizes clinicas como instrumento de melhoria da qualidade da assistência complementar: o papel da Agência Suplementar de Saúde. Dissertação (Mestrado em Saúde Pública) - Escola Nacional de Saúde Pública, Fiocruz, Rio de Janeiro, 2003.

SAUNDERS, R.P. et al. Evaluation and Development of Potentially Better Practices for Improving Family-Centered Care in Neonatal Intensive Care Units. Pediatrics, v. III, n. 4, p. e437, 2003.

SHIELDS, L.; TANNER, A. Pilot study of a tool to investigate perceptions of FCC in different care settings. Pediatric Nurs., v. 30, n. 3, p. 189-97, May/Jun 2004.

STEWART, D.L. Vermont Oxford Network: effective and efficient medical care for the neonate. Jky Med Assoc., v. 99, n. 2, p. 65-7, Feb 2001.

VIANNA, C.M.M.; CAETANO, R. Avaliação Tecnológica em Saúde: introdução a alguns conceitos básicos. [Texto didático preparado para o Mestrado Profissional em Administração em Saúde: Disciplina de Gestão Tecnológica] Ministério da Saúde/IMS-UERJ, 2001.

WARD, F.R. Parents and professionals in the NICU: communication within the context of ethical decision making-an integrative review. Neonatal Network, v. 24, n. 3, p. 25-33, May/Jun 2005.

\section{Nota}

1 Grupos de profissionais de saúde, de diferentes centros mundiais de terapia intensiva, que trabalham com objetivo de realizar estudos colaborativos em neonatologia. 
Humanized care for low birth weight newborns. The Kangaroo Care Method and family-centered care: correspondence and specificities

The main purpose of this paper is to introduce the Human Attention of Low Birth Weight-Kangaroo Care as clinical guidelines in neonatal intensive care. It will also try to link this clinical guideline and another component of neonatal infant care: the Family-Centered Care. Based on this relationship, this paper proposes that Human Attention of Low Birth Weight-Kangaroo Care should be catalogued as a Potentially Better Practices and also, considered as a Health Care Technology.

> Key words: Kangaroo Mother Care; family-centered care; low birth weight; clinical guidelines; health technologies; potentially better practices. 